\title{
Editorial
}

\section{Meus Dados, Minha Vida: Objetivos Individuais e Papel da Comunidade de Pesquisadores na Área de Negócios}

My Data, My Life: Individual Goals and Role of the Business Research Community

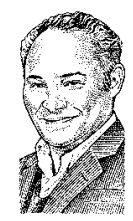

Wesley Mendes-Da-Silva ${ }^{1,2}$

As pressões sobre indivíduos que desempenham o papel de pesquisadores, para que esses contribuam para a produção de conhecimento, constituem um tópico de elevado interesse (Silva, 2019). Os impactos da avaliação de desempenho, nos níveis individual e institucional, são inúmeros e invariavelmente implicam na capacidade de progressão profissional, assim como acessar recursos para pesquisa. Em função da percepção de que a produção de resultados de pesquisa pode definir o futuro profissional de indivíduos e/ou a consolidação de instituições de pesquisa, constata-se, em alguma medida, a busca crescente por maior quantidade de artigos científicos. Contudo, existe em paralelo crescente preocupação com a produção de pesquisa de maior impacto. Ao redor dessas questões, um rol expressivo de aspectos relevantes ganha espaço.

A esse respeito, a transparência dos procedimentos adotados para conduzir a pesquisa (Aguinis, Ramani, \& Alabduljader, 2018; Mendes-Da-Silva, 2018), a capacidade de reproduzir resultados (Kousta, Pastrana, \& Swaminathan., 2019), o compartilhamento de dados, a disponibilização de códigos utilizados na investigação científica, e outros fatores,

\footnotetext{
'Fundação Getulio Vargas, Escola de Administração de Empresas de São Paulo, São Paulo, SP, Brasil.

${ }^{2}$ Editor-chefe da RAC - Revista de Administração Contemporânea.
}

apresentam-se merecedores de atenção da comunidade acadêmica. Seja qual for o ângulo a partir do qual seja observado esse contexto, convém destacar que os avanços na área de tecnologia, na disponibilidade de dados, assim como na capacidade de processamento, apontam para uma imperativa mudança de atitude em relação à disponibilização de dados e outras informações relevantes acerca do processo da pesquisa, sobretudo em nome da transparência (Mendes-Da-Silva, 2019a).

Organismos internacionais, como Council of Science Editors (CSE) têm apontado a prevalência de preferências individuais, em detrimento do progresso acelerado do conhecimento científico. Em outras palavras, tem sido constatada reduzida disposição à promoção da transparência na pesquisa, com potenciais prejuízos à criação de conhecimento, tendo em vista o julgamento de que a retenção de dados e demais procedimentos podem constituir vantagem competitiva de profissionais pesquisadores na busca por crescimento profissional em diversas áreas de conhecimento.

Como citar: Mendes-da-Silva, W. (2020). Meus dados, minha vida: Objetivos individuais e papel da comunidade de pesquisadores na área de negócios. Revista de Administração Contemporânea, 24(2), 197-200. https://doi.org/10.1590/1982-7849rac2020190403 
Segundo palavras de Erlandsson (2017, p. 157):

Brazil ranks 24th among countries that publish the most in high-impact journals, according to Nature Index. This fact highlights the potential for scientific production in the country. However, the dissemination of research data is unfortunately not always the main motivation behind this production. Many authors publish to strengthen their chances of receiving support from funding agencies or to advance in their academic careers (or both) and consequently focus on increasing their number of published articles to the detriment of quality. (Grifo nosso).

Essa é uma questão que parece ser merecedora de reflexão por parte da comunidade acadêmica, vis $a$ vis seu papel na sociedade. Revistas científicas e editores, conforme visão de Schultz (2019), têm papel essencial para ajudar impor níveis apropriados e consistentes de transparência, tanto em pesquisa publicada, como ao longo do processo de revisão e publicação. Maior transparência pode ser decisiva para expor problemas, tanto não intencionais, como maliciosos, ajudar na reprodução/replicação, além de contribuir para aumentar a confiança da sociedade na produção de conhecimento oferecida pela comunidade de pesquisadores via práticas de Ciência Aberta (Martins, 2019).

Os periódicos científicos, para além das funções historicamente conhecidas, têm sido frequentemente considerados gatekeepers de dados e de informações. Uma das maiores responsabilidades, em todos os níveis, é realçar e manter accountability no âmbito da pesquisa disseminada. Os campos científico e tecnológico estão se tornando mais diversificados, não estando mais restritos ao meio dos indivíduos pesquisadores. Os periódicos científicos podem desempenhar seu papel para ajudar a garantir que a comunicação científica possa efetivamente refletir essa mudança (Parker, 2019). A RAC, em particular, tem adotado uma política de dados abertos mediante a qual os autores são convidados a compartilhar dados e códigos, tendo permitido a publicação dos primeiros artigos com dados, materiais, e/ou códigos abertos, os quais estão centralizados em repositório público: https://dataverse.harvard.edu/dataverse/rac/ (recuperado em 09 de Dezembro, 2019). Além disso, foi criada uma editoria dedicada às rotinas típicas dessa modalidade de compartilhamento de modo a acelerar a produção de conhecimento, além de colaborar para assegurar níveis de transparência na produção de conhecimento (Martins, 2019).

$\mathrm{O}$ esforço de pesquisadores para construir dados de elevada qualidade é algo a ser devidamente reconhecido. Ademais, a RAC assume que os autores de trabalhos assumem a responsabilidade pela publicação, assegurando que os dados sejam precisos, que todos os autores merecedores tenham sido devidamente creditados, e que todos os autores tenham aprovado o conteúdo final. Em adição, tendo em vista a comunicação de conhecimento gerado (Mendes-Da-Silva, 2019b), os autores ainda acumulam a responsabilidade de responder às eventuais perguntas e questões após a publicação do manuscrito. Nesse sentido, a publicação conjunta de resultados de pesquisa juntamente com os dados utilizados, além de permitir o atendimento a práticas transparentes, permite que os autores mantenham facilitado o resgate da memória de seu trabalho em oportunidades futuras. Quem de nós, após alguns anos, é capaz de facilmente localizar dados e memória de cálculo de trabalhos publicados?

Em termos de reconhecimento da contribuição individual de autores, graças a avanços tecnológicos já é possível encontrar ambientes virtuais dedicados a publicamente reconhecer as contribuições de autores. Conforme destacam Allen, O'Connell e Kiermer (2019), Brand, Allen, Altman, Hlava e Scott (2015) introduzem a CRediT - Contributor Role Taxonomy https://www.casrai.org/credit.html (recuperado em 09 de Dezembro, 2019), segundo a qual é proposta uma mudança para uma maneira holística de descrever as contribuições para a produção acadêmica publicada, indo além de um conceito estático de autoria. Esse artigo recomenda o uso de uma taxonomia simples, mas abrangente, que pode ser usada por autores ao enviar um artigo para publicação, a fim de permitir que o alcance e a natureza das contribuições para a produção acadêmica publicada fossem capturados de forma transparente, e mediante formato estruturado. A esse respeito têm surgido alternativas, como a que é encontrada em: $<$ https://rescognito.com/about.php> (recuperado em 09 de Dezembro, 2019).

Conforme entendimento de Kousta, Pastrana e Swaminathan (2019), embora os editores e editores de periódicos devam desempenhar seu papel na promoção da transparência e da reprodutibilidade, o impacto significativo e sustentado que reforça as práticas de pesquisa aberta por meio de orientação, treinamento e processo de pesquisa são dependentes de várias partes interessadas. As instituições e financiadores, em particular, precisarão prover o suporte necessário em termos de treinamento, orientação e infraestrutura, incluindo recursos e suporte para gerenciar os resultados subjacentes da pesquisa, dados, código, materiais e protocolos. A RAC tem assumido que as práticas de Ciência Aberta, incluindo-se a disponibilização de dados e códigos utilizados na pesquisa, é um aspecto em evolução. Portanto, há trabalho a ser feito, e certamente a mudança de cultura na coleta, uso, e reuso dos dados é parte do desafio a ser vencido. 


\section{REFERENCIAS}

Aguinis, H., Ramani, R. S., Alabduljader, N. (2018). What you see is what you get? Enhancing methodological transparency in management research. Academy of Management Annals, 12(1), 83-110. https://doi.org/10.5465/annals.2016.0011

Allen, L., O'Connell, A., \& Kiermer, V. (2019). How can we ensure visibility and diversity in research contributions? How the Contributor Role Taxonomy (CRediT) is helping the shift from authorship to contributorship. Learned Publishing, 32(1), 71-74. https://doi.org/10.1002/leap.1210

Brand, A., Allen, L., Altman, M., Hlava, M., \& Scott, J. (2015). Beyond authorship: Attribution, contribution, collaboration, and credit. Learned Publishing, 28(2), 151- 155. https://doi.org/10.1087/20150211

Erlandsson, B. (2017). Enrolling brazilian editors in CSE's certificate program: A successful initiative. Science Editor, 40(2), 157-158. Retrieved from https://www.csescienceeditor.org/article/enrolling-brazilian-editors-cses-certificate-program-successful-initiative/

Kousta, S., Pastrana, E., Swaminathan, S. (2019). Three approaches to support reproducible research. Science Editor, 42(3), 77-82. Retrieved from https://www.csescienceeditor.org/article/three-approaches-to-support-reproducible-research/

Martins, H. (2019). A importância da ciência aberta (open science) na pesquisa em Administração. Revista de Administração Contemporânea, 24(1). https://doi.org/10.1590/ 1982-7849rac2020190380

\section{Autor}

\section{Wesley Mendes-Da-Silva*}

Rua Itapeva, 474, $8^{\circ}$ andar, 01332-000, São Paulo, SP, Brasil.

E-mail: rac.wesley.mendes@gmail.com

https://orcid.org/0000-0002-5500-4872

(10* Autor Correspondente

\section{Corpo Editorial Científico e Equipe Editorial para esta edição:}

\section{Conselho Editorial}

Anielson Barbosa da Silva (UFPB, João Pessoa, Brasil)

Antonio Carlos Gastaud Maçada (UFRGS, Porto Alegre, Brasil)

Ely Laureano Paiva (FGV, São Paulo, Brasil)

Fabio Vizeu Ferreira (UP, Curitiba, Brasil)

Maria José Tonelli (FGV, São Paulo, Brasil)

Rogério Hermida Quintella (NPGA/UFBA, Salvador, Brasil)

Valmir Emil Hoffmann (UnB, Brasília, Brasil)

Wesley Mendes-da-Silva (EAESP/FGV, São Paulo, Brasil)

\section{Editor-chefe}

Wesley Mendes-da-Silva (EAESP/FGV, São Paulo, Brasil)
Mendes-Da-Silva, W. (2018). Promoção de transparência e impacto da pesquisa em negócios. Revista de Administração Contemporânea, 22(4), 639-649. https://doi.org/10.1590/ 1982-7849rac2018180210

Mendes-Da-Silva, W. (2019a). Temos sido transparentes o suficiente? Desafios à replicabilidade e à credibilidade da pesquisa na área de negócios. Revista de Administração Contemporânea, 23(5). https://doi.org/10.1590/ 1982-7849rac2019190306

Mendes-Da-Silva, W. (2019b). Convergência, comunicação, e impacto da pesquisa em negócios. Revista de Administração Contemporânea, 23(1). https://doi.org/10.1590/ 1982-7849rac2019180346

Parker, C. A. (2019). The editor's role in avoiding gender bias. Science Editor, 42(3), 85-86. Retrieved from https://www. csescienceeditor.org/article/the-editors-role-in-avoiding-gender-bias/

Schultz, J. (2019). Stop, collaborate, and listen: Working together to enhance a scientific system under pressure. Science Editor, 42(3), 74-76. Retrieved from https://www. csescienceeditor.org/article/stop-collaborate-and-listen-working-together-to-enhance-a-scientific-system-under-pressure/

Silva, A. B. da (2019). Produtivismo acadêmico multinível: Mercadoria performativa na pós-graduação em administração. Revista de Administração de Empresas, 59(5), 341-352. http://dx.doi.org/10.1590/S0034-759020190504

\section{Editores Associados}

André Luiz Maranhão de Souza-Leão (UFPE, Recife, Brasil) Fabio Caldieraro (EAESP/FGV, São Paulo, Brasil) Gilnei Luiz de Moura (UFSM, Santa Maria, Brasil) Henrique Castro Martins (IAG PUC-Rio, Rio de Janeiro, Brasil) Ismael Ali Ali (Kent State University, Ohio, EUA)

Marcus Cunha Junior (University of Georgia, EUA)

Paulo César Matui (UniGranRio, Rio de Janeiro, Brasil) Samy Dana (FGV/EAESP, São Paulo, Brasil)

Victor Manoel Cunha de Almeida (COPPEAD/UFRJ, Rio de Janeiro, Brasil)

\section{Corpo Editorial Científico}

Aureliano Angel Bressan (CEPEAD/UFMG, Belo Horizonte, Brasil)

Bryan Husted (York University, Canadá)

Carlos M. Rodriguez (Delaware State University, EUA)

Cristiana Cerqueira Leal (Universidade do Minho, Portugal)

Diógenes de Souza Bido (Mackenzie, São Paulo, Brasil)

Elin Merethe Oftedal (University of Tromsø, Noruega)

Emilio Jose Monteiro Arruda Filho (Unama, Belém, Brasil)

Fábio Frezatti (FEA/USP, São Paulo, Brasil)

Felipe Monteiro (Wharton/University of Pennsylvania, EUA)

Howard J. Rush (University of Brighton, Reino Unido)

James Robert Moon Junior (Georgia Institute of Technology, EUA)

John L. Campbell (University of Georgia, EUA)

José Antônio Puppim de Oliveira (United Nations University, Yokohama, Japão) Julián Cárdenas (Freie Universität, Berlin, Alemanha) 
Lucas Barros (EAESP/FGV, São Paulo, Brasil)

Luciano Rossoni (UniGranRio, Rio de Janeiro, Brasil)

M. Philippe Protin (Université Grenoble Alpes, França)

Paulo Estevão Cruvinel (Embrapa Instrumentação, São Carlos, Brasil)

Rodrigo Bandeira de Mello (Merrimack College, EUA)

Rodrigo Verdi (MIT Massachusetts Institute of Technology, Cambridge, EUA)

Valter Afonso Vieira, (UEM, Maringá, Brasil)

Wagner Kamakura (Jones Graduate School of Business, Rice University, Houston, EUA)

\section{Editoração}

Diagramação e Normas da APA: Kler Godoy (ANPAD, Maringá, Brasil)

Periodicidade: Bimestral.

Circulação: Acesso totalmente gratuito. 